\title{
A wearable fiber-optic sensor for monitoring human elbow and wrist joint motion
}

\author{
Min $\mathrm{Li}^{\mathrm{a}, \mathrm{b} *}$, Bo He $\mathrm{He}^{\mathrm{a}}$, Xingang Zhao ${ }^{\mathrm{b}}$, Jun Xie ${ }^{\mathrm{a}}$, Wei $\mathrm{Yao}^{\mathrm{c} *}$, Guanghua $\mathrm{Xu}^{\mathrm{a}}$ \\ a Department of Mechanical Engineering, Xi'an Jiaotong University, Xi'an 710049, \\ China
}

b State key Laboratory of Robotics, Shenyang Institute of Automation, Chinese Academy of Sciences, Liaoning 110016, China.

c Department of Biomedical Engineering, University of Strathclyde, Glasgow G4 ONW, $U K$

*corresponding author:

$\operatorname{Min} \mathrm{Li}$

Department of Mechanical Engineering, Xi'an Jiaotong University, Xi'an 710049, China.min.li@mail.xjtu.edu.cn

Wei Yao

Department of Biomedical Engineering, University of Strathclyde, Glasgow G4 0NW, UK.w.yao@strath.ac.uk

Min Li, Bo He, Xingang Zhao, Jun Xie, Wei Yao \& Guanghua Xu (2020) A wearable fiber-optic sensor for monitoring human elbow and wrist joint motion, Advanced Robotics, DOI: 10.1080/01691864.2020.1837671 


\section{A wearable fiber-optic sensor for monitoring human elbow and wrist joint motion}

This article describes a wearable sensor for monitoring the motion of human elbow and wrist joints. The sensor converts the joint motion angle into a displacement, which is monitored using a fiber-optic sensor based on reflective light intensity modulation. A mathematical model is established, and the potential influencing factors of the sensor are investigated. Two prototypes for elbow and wrist joint angle monitoring are fabricated and validated. The experimental results indicate that the proposed sensor has a measurement range of $0-60^{\circ}(0-$ $\left.120^{\circ}\right)$, with a resolution of $0.338^{\circ}\left(3.6^{\circ}\right)$ for monitoring the wrist joint palm flexion (elbow joint flexion). The proposed sensor has the potential to be used for rehabilitation purposes and monitoring of joint angles while performing reaching tasks, which are the most common upper-limb gestures.

Keywords: fiber-optic sensor; joint angle monitoring; light intensity-modulated sensor; wearable sensor

Subject classification codes: include these here if the journal requires them

\section{Introduction}

Many medical applications can benefit from continuous monitoring of human joint movement. For example, motor rehabilitation and assistance require a quantitative assessment of the performed tasks by monitoring joint motion [1]. Many wearable soft robots for motor rehabilitation and assistance are not equipped with sensors to monitor either the robot motion or the joint angle motion of the patient, making it difficult to control the robot precisely [2-5].

Measuring human joint angles with electrogoniometers is a standard method used by physiotherapists to evaluate and score the performance of patients who perform typical tasks [6-7]. Electrogoniometers require the user to measure and read the data manually. Despite their widespread usage, electrogoniometers require careful sensor alignment, may restrict movements and are inconvenient to use. Electromagnetic 
motion monitoring systems are also widely used [8-9]. This kind of sensing mechanism requires a magnetic field generator and is susceptible to distortion of the magnetic field. Thus, it has strict requirements for the monitoring environment. No metal should not be onsite during the measurement process. Camera-based motion capture systems such as the VICON system are also often used to monitor joint motion. However, these are expensive systems that can only be used inside a laboratory environment. Moreover, they often require markers on the body, and video motion analysis is time-consuming [10-11]. Depth sensors, such as the Kinect, are less expensive options [12]. However, depth sensors are not applicable to some human postures with body occlusion. Another approach is wearable bending sensors, which are usually made from stretchable electronic materials, such as variable resistors, piezoelectric materials, and electroactive polymers [13-19]. These skin-mountable and wearable sensors, which are attached to the fabric, can provide repeatable results and allow for the simultaneous measurement of multiple joints and information storage in a database. However, some of the sensor characteristics require improvements, such as the response time, stability, and sensitivity [20].

Compared to the above sensing mechanisms, fiber-optic-based approaches have several advantages, such as immunity to electromagnetic interference, ease of fabrication, temperature insensitive, low weight, ruggedness, and low cost [21,22]. Optical fiber sensors can be divided into three categories: intensity-modulated, phasemodulated, and wavelength-modulated. Intensity-modulated optical fiber sensors are used in a wide variety of applications because they have low complexity, low-cost, are easy to fabricate, and are temperature-insensitive [23]. The light loss can be associated with attenuation based on microbending and macrobending. Optical fibers are wrapped around the body, and light loss occurs due to a change in the curvature radius of the 
optical fiber when the joint angle increases. The change in the joint angle can be indirectly measured by the loss of light intensity [24-25]. This type of sensor has a small measurement range and is difficult to adapt to large joint motion [25]. Another type of intensity-modulated sensor is a reflective optical fiber sensor. This sensor consists of a light emitter, two fibers, a reflective surface, and a light detector. The light from the end of an incident fiber (IF) is reflected by the reflective surface and is collected by a receiving fiber $(\mathrm{RF})$. The displacement between the end of the fibers and the reflective surface varies because of the influence of external factors, such as the contact force, which can be measured by a change in the light intensity. This sensing mechanism has been successfully applied to measure small displacement [26-28], force [29], torque [30], contact [31], and tactile array information [32]. This sensing mechanism may have potential for applications in joint angle monitoring if the sensor can be modified to measure a large displacement associated with a change in the joint angle.

This paper describes the design and characterization of a wearable, lightweight fiber-optic sensor based on reflective light intensity modulation for elbow and wrist motion angle monitoring. The sensor requirements, design concepts, and the mathematical models of the sensing principle are described in Section 2. After analyzing the key influencing factors on the sensor performance in Section 3, the design, manufacturing, and validation of the prototypes for elbow and wrist joint angle monitoring are described in Section 4. Section 5 provides the conclusions.

\section{Methodology}

\subsection{Sensor requirement and design concept}

Sensors for human joint angle determination should not interfere with the joint motion. Wearability, comfort, small size, low weight, high efficiency, safety, flexibility, and 
easy integration into rehabilitation robots are essential factors that should be considered when designing sensors for human joint angle monitoring. Most activities in daily life require elbow joint bending angles between $30^{\circ}$ and $130^{\circ}$, and the wrist joint has a range between $15^{\circ}$ and $60^{\circ}$ when a person is sitting and performing typical close-range motions [25]. In our design, the maximum measurable angle of the elbow sensor was set to $120^{\circ}$. Although wrist movements include palm flexion, dorsiflexion, radial deviation, and ulnar deviation, only palm flexion was considered in this study. Since the range of palm flexion is larger than that of dorsiflexion, the sensor can also be assembled on the other side of the forearm to monitor dorsiflexion. The maximum measurable palm flexion for the wrist sensor was set to $60^{\circ}$. According to Nitschke et al. [33], the mean error limit for reliable measurements in the evaluation of movement impairments in clinical contexts is $5^{\circ}$. Here, the target resolution of the elbow angle sensor was $4^{\circ}$, and the target resolution of the sensor for measuring the wrist palm flexion angle was $1^{\circ}$.

As shown in Figure 1 (a), points $A_{l}$ and $B_{l}$ represent two fixed points on the skin surface near the elbow joint, and $d_{1}$ is the distance between the two points. When the elbow joint moves from the position represented by the dashed line to the position represented by the solid line, point $B_{1}$ moves to the location indicated by point $C_{l}$. This action increases the distance between $\mathrm{A}_{1}$ and $\mathrm{B}_{1}$ on the skin surface by $\Delta d_{1}$. The elbow joint angle can be indirectly measured by monitoring the displacement $\left(\Delta d_{l}\right)$. The wrist palm flexion angle can also be monitored using a similar principle (shown in Figure 1(b)). [figure 1 near here]

\subsection{Relationship between the joint angle and the displacement}

As illustrated in Figure 2 (a), an experiment was conducted to evaluate the relationship between the elbow angle and the displacement $\Delta d$. A non-retractable cable was fixed to the subject's upper arm via a 3D printed structure, Nylon straps, and a bolt. The cable 
was limited to moving along the posterior side of the elbow using suture knots. A semicircular protractor was used to measure the joint angle (see Figure 2 (a)). The acromion, the lateral epicondyle of the humerus, and the center of the back of the wrist were used to determine the elbow joint angle. Nine participants were selected for the experiment, including 3 females and 6 males from 22 to 25 years old. During the test, their elbows moved from $0^{\circ}$ to $130^{\circ}$, and the displacement $\Delta d$ was measured at intervals of $10^{\circ}$. The test was repeated three times. This study was approved by the institutional review board of Xi' an Jiaotong University. Participants signed a written consent form before conducting the experiments that were part of this study. The experimental data are shown in Figure 2 (a). Linear fitting was performed for the elbow angle and the displacement $\Delta d$ as follows:

$$
\Delta d_{1}=0.4835 \theta_{1}+3.7
$$

where $\theta_{l}$ is the angle of the elbow movement. There was a linear relationship between the displacement and the elbow joint angle $\left(R^{2}=0.9053\right)$. When the elbow was bent $130^{\circ}$, the displacement was $66.618 \mathrm{~mm}$. The measurement range was extended by $10 \%$ to meet the needs of most people for practical applications. Our ultimate target for the measurable displacement was $73 \mathrm{~mm}$. The slope of the fitted curve for all participants was between 0.324 and 0.642 .

We conducted a similar experiment for the wrist joint. The experimental set-up and results are shown in Figure 2 (b). Linear fitting was performed for the wrist palm flexion angle and the displacement $\Delta d$ :

$$
\Delta d_{2}=0.2759 \theta_{2}+0.9598
$$

where $\theta_{2}$ is the palm flexion angle, and $\Delta d_{2}$ is the displacement $\left(R^{2}=0.9245\right)$. The displacement was $16.43 \mathrm{~mm}$ (Eq. (2)) when the wrist was bent at $60^{\circ}$. After extending 
the range by $10 \%$, the measurement range was $0-18 \mathrm{~mm}$. The slope of the fitted curve for all participants was between 0.225 and 0.338 . [figure 2 near here]

\subsection{Mathematical modeling of the reflective intensity-modulated optical fiber sensor}

The intensity-modulated optical fiber sensor acquires measurements using a pair of straight parallel optical fibers integrated into a longitudinally moving reflective surface to modulate the reflected optical signal intensity. As shown in Figure 3, light is transmitted from a light source to the tip of the IF, and the light is reflected by the reflective surface facing the tip of the fiber. Part of the reflected light is transmitted to the light detector through the RF. The light intensity at the RF is affected by the distance $(h)$ between the reflective surface and the fiber tip. Therefore, the displacement can be monitored by detecting the light intensity change. This sensing method was proved to be practical for small displacement measurements [34-35]. However, joint angle monitoring requires sensing of larger displacement than other applications. Therefore, mathematical modeling was conducted to provide theoretical guidance for the sensor design. [figure 3 near here]

The configuration had the following characteristics, and some assumptions were made:

(1) The power of the light source was constant. The end surfaces of the two fibers were on the same plane. In addition, the axes of the two fibers were parallel.

(2) The reflective surface was a reflective sticker.

(3). The reflective surface was perpendicular to the axis of the fiber and moved in the axial direction of the fiber without tilting. 
(4). The light emitted by the IF formed a symmetrical cone of light with a divergence angle $\theta$, and all light energy was contained in the cone. In addition, the end surface of the RF was on the bottom surface of the light cone.

The light energy received by the RF was determined by calculating the overlap area between the bottom surface of the light cone (BSLC) and the end surface of the RF $[34,35]$. The distance between the reflective surface and the fiber $(h)$ was the measurement target. In Figure 3, $z$ represents the height of the virtual light cone, and $h_{1}$ represents the distance between the apex of the light cone and the IF. Therefore, $h=(z-$ $\left.h_{1}\right) / 2 . \theta$ is half the taper angle of the light cone. $\omega$ is the radius of the bottom surface of the light cone. Hence, $\omega=z / \tan \theta . a$ represents the distance between the center of the IF and the RF.

As shown in Figure 4, when $z<(a+r) / \tan \theta$, the light cone and the RF are separated. The overlap area and the received light energy are both zero. When $(a+3 r) / \tan \theta<z$, the overlap area is the area of the RF. $r$ denotes the radius of the optical fiber core. When $(a+r) / \tan \theta<z<(a+3 r) / \tan \theta$, the overlap area $\left(S_{2}\right)$ can be calculated as:

$$
\begin{gathered}
\alpha_{2}=\arccos \frac{r^{2}+(a+2 r)^{2}-\omega^{2}}{2 r(a+2 r)} \\
y=a+2 r-r \cdot \cos \alpha_{2} \\
x=\sqrt{\omega^{2}-y^{2}} \\
\alpha_{1}=\arccos \frac{y}{\omega} \\
S_{21}=\frac{1}{2} \alpha_{1} \omega^{2}-\frac{1}{2} x y \\
S_{22}=\frac{1}{2} \alpha_{2} r^{2}+\frac{1}{2} x(y-2 r-a)
\end{gathered}
$$




$$
S_{2}=2\left(S_{21}+S_{22}\right)
$$

[figure 4 near here]

The geometric relationships between $S_{21}, S_{22}, \alpha_{1}$, and $\alpha_{2}$ are shown in Figure 5. In addition, $(x, y)$ denotes the coordinates of the intersection of the BSLC and the RF.

[figure 5 near here]

When the height of the virtual light cone changes, the overlap area is calculated as follows:

$$
S=\left\{\begin{array}{cl}
0, & z<\frac{a+r}{\tan \theta} \\
2\left(S_{21}+S_{22}\right), & \frac{a+r}{\tan \theta}<z<\frac{a+3 r}{\tan \theta} \\
\pi r^{2}, & \frac{a+3 r}{\tan \theta}<z
\end{array}\right.
$$

The light intensity is calculated using Equation (11) when the light source is a Gaussian beam:

$$
I(\rho, \omega(h))=\frac{2 P_{e}}{\pi \omega(h)^{2}} \exp \left(-\frac{2 \rho^{2}}{\omega(h)^{2}}\right)
$$

where $P e$ is the total light power at the bottom surface of a single light cone, $\omega(h)$ is the radius of the cross-section when the distance between the reflective surface and the fiber is $h$, and $\rho$ is the distance between the measurement point and the axis of the cone.

When the light source is a Gaussian beam, the theoretical light intensity $(P)$ received at the detector for a distance of $h$ is:

$$
P(h)=I(\rho, \omega(h)) \cdot S
$$

The relationship between the light intensity and the distance $h$ is shown in Figure 6 for $r=0.2425 \mathrm{~mm}$ and $a=3.16 \mathrm{~mm}$. [figure 6 near here] 
Equations (10)-(12) and Figure 6 show that the received light intensity changes with $h$ when the distance between the two fibers and the source of the light are fixed. The curve depicting the relationship between the light intensity and $h$ can be divided into three parts: no response, increase in light intensity, and decrease in light intensity. In the no response zone, the sensor cannot detect any light. The portion of the curve that increases is characterized by a high rate of increase. Here, we focus on the portion of the decrease in light intensity because the low rate of decrease provides a wide measurement range. According to the mathematical model, for the design of the sensor structure, the fiber core diameter, the distance between the IF and the RF, and the stability of the light source should be considered.

\subsection{Sensor structure}

The diagram of the designed sensor structure is shown in Figure 7. The sensor contains a light source, a light detector, an IF, an RF, a sensor tube, a non-retractable cable, and a cable fixture. Inside the sensor tube, there is a fiber fixture to ensure that the IF and RF are parallel, a sliding part, two springs linking the sliding part and the tube, and a reflective surface that is attached to the other of the sliding part. The sensor tube acts as a guide rail to ensure that the sliding part remains parallel to the end surface of the fiber during movement. One end of the non-retractable cable is attached to the sliding part, and the other end is attached to the cable fixture located on the person's arm. The cable is limited to movement along the skin of the joint. When the joint is bent, the cable fixture moves, pulling the sliding block at the other end of the cable away from the end surface of the two fibers. The springs ensure that the sliding block returns to the original position when the joint moves to the original position. Therefore, the distance $h$ and the light intensity received by the detector are changed. [figure 7 near here] 


\section{Analysis of influencing factors}

The factors that may influence the sensor performance were investigated in comparison experiments. The experimental setup is shown in Figure 8. The sensing module was fixed to one side of the motor powered linear module and connected to the KEYENCE sensor (FSN11MN, KEYENCE CO., Japan) through two optical fibers (an IF and an RF). The KEYENCE sensor was used as both a light source (red light with a wavelength of $630 \mathrm{~nm}$ ) and a detector. It had a repeatability of $\pm 0.5 \%$, and the response time was less than $5 \mathrm{~ms}$. A reflective surface was fixed on the bottom of the sliding part. The end of the sliding part was fixed to linear slider. A stepper motor provided accurate displacement input to the reflective surface by dragging the sliding part moving inside the sensor tube. A data acquisition card (National Instruments6210) was used to record voltage from the KEYENCE sensor at the frequency of $1 \mathrm{kHz}$. The farther the distance between the end face of the fibers and the reflective surface, the lower the voltage that the data acquisition recorded. By comparing the voltage outputs to the displacement inputs, the characteristic curve of the sensor can be obtained. [figure 8 near here]

\subsection{Influence of the light transmittance of the tube}

Two sets of experiments were used to investigate the influence of the light transmittance of the tube on the sensor performance. The diameters of the tubes in the two sets of experiments were $19.57 \mathrm{~mm}$ and $15.74 \mathrm{~mm}$. The light transmittance of the sensor tube was changed by wrapping it with black tape, as shown in Figure 9 (a). The stroke of the stepper motor was $50 \mathrm{~mm}$. The linear slider moved at a constant speed of $5 \mathrm{~mm} / \mathrm{s}$ to move the sliding part backward and forward. Each set of experiments was repeated 5 times. The normalized data are shown in Figure 9 (b) and Figure 9 (c). The result revealed that ambient light had little effect on the light intensity. [figure 9 near here] 


\subsection{Effect of the distance between the two fibers}

Equations 10-12 indicate that the distance between the two fibers is a significant factor affecting other parameters of the displacement sensor, including the area of no response in the light intensity curve, the position of the peak, and the linearity of the curve in the area of decrease. The following test was designed to investigate the effect of the distance between the two fibers on the sensor performance. The tube's diameter was $19.57 \mathrm{~mm}$. The measured distances between the two fibers were $5.20 \mathrm{~mm}, 6.01 \mathrm{~mm}$, $6.97 \mathrm{~mm}, 7.95 \mathrm{~mm}$, and $9.92 \mathrm{~mm}$. The fiber was Grade SH-4001 (outer diameter: 2.2 $\mathrm{mm}$, core diameter: $1 \mathrm{~mm}$ ). During the test, the position of the fiber-optic sensor was fixed, and the fiber fixture was moved to change the distance. As shown in Figure 10, as the distance increased, the peak moved to the left, and the linearity of the area of decrease of the curve increased. The experimental results revealed that the performance of the sensor improved as the distance increased. [figure 10 near here]

\subsection{Influence of the tilt angle of the reflective surface}

It is challenging to keep the reflective surface and the optical fibers parallel due to manufacturing difficulties and relative motion during use. Hence, it is necessary to investigate the influence of the tilt angle of the reflective surface on the sensor performance. During the test, the angle between the reflective surface and the surface of the optical fiber ranged from $0^{\circ}$ to $5^{\circ}$, with an interval of $1^{\circ}$. A tube with an internal diameter of $19.57 \mathrm{~mm}$ was used. The sliding part was replaced in the experiment to change the tilt angle. Data with a movement range of $50 \mathrm{~mm}$ was measured and recorded. The normalized results are shown in Figure 11. The tilt angle had no significant effect on the measurement results. In other words, the angle between the reflective surface and the surface of the fibers does not have to set to a specific angle in 
the manufacturing and assembly process. [figure 11 near here]

\section{Prototyping and validation}

\subsection{Sensor prototyping and displacement sensing}

Prototypes of two sensors (wrist and elbow) for joint motion monitoring were developed.

\subsubsection{Sensor for monitoring wrist joint motion}

The fabricated prototype for measuring the wrist joint motion is shown in Figure 12. An FS-N11MN (KEYENCE CO., Japan) was used as both a light source and a detector. The FS-N11MN had a light source consisting of a red light-emitting diode with a wavelength of $630 \mathrm{~nm}$. It had a repetition accuracy of $\pm 0.5 \%$, and the response time was less than $5 \mathrm{~ms}$. The sensor tube, the fiber fixture, the cable fixture, and the sliding part were made from VisiJet Crystal using a rapid prototyping machine (3D Systems MJP3600). The reflective surface was a reflective sticker (3M 610 Series, PET/Acrylic), which was attached to the sliding part. One pair of polymer optical fibers LXH0501$10(\mathrm{E})$, with a core diameter of $0.25 \mathrm{~mm}$, a diameter with a jacket of $1 \mathrm{~mm}$, a reflective index of 1.49 , and a numerical aperture of 0.50 were used for transmitting and receiving light. As shown in Equation (2), the displacement was $18 \mathrm{~mm}$, when the wrist was bent at $60^{\circ}$. Therefore, the sliding distance of the sensor was set to $18 \mathrm{~mm}$.

The resolution of the KEYENCE was set to $0.01 \mathrm{~V}$. The slope of the output curve of the sensor $\left(K_{W_{-} \min }\right)$ was calculated as follows to ensure that the resolution of the wrist angle sensor was $1^{\circ}$ :

$$
K_{W_{-} \min }>\frac{0.01}{1 * Q},
$$


where $Q$ is the minimum slope of the fitted curve of the subjects' wrist motion angle and the displacement. According to the experimental results presented in Section 2.2, $Q$ was 0.225 . Therefore, $K_{W_{-} \min }$ was 0.044 . Preliminary tests indicated that the slope of the output curve of the sensor was larger than 0.044 when the optical fiber spacing was greater than $6 \mathrm{~mm}$. In our design, the distance between the two fibers was $6.31 \mathrm{~mm}$. The KEYENCE sensor was adjusted to the MEGA mode with a power setting of 64 (The power setting can range from 1 to 100). [figure 12 near here]

The testbed depicted in Figure 8 was used to investigate the displacement sensing performance of the designed sensor. The reflective surface was connected to the stepper motor by thin rods rather than the cable to obtain an accurate result of the relationship between the optical fiber intensity and displacement. The linear slider moved at a constant speed of $5 \mathrm{~mm} / \mathrm{s}$ to drive the sliding part backward and forward. Each set of experiments was repeated 5 times. As shown in Figure 13 (a), the sensor provided an excellent and repeatable output. As shown in Figure 13 (b), when the displacement was in the range of 7.4-50 $\mathrm{mm}$, the monotonicity of the voltagedisplacement curve was consistent. When the displacement was in the range of 7.9$27.9 \mathrm{~mm}$ (Figure $13(\mathrm{c})$ ), the slope was between -0.04469 and -0.2479 .

\subsubsection{Sensor for monitoring elbow joint motion}

The sensor structure and the prototyping process was similar for the elbow joint. The fiber used in the sensor for measuring the elbow motion angle was SH-4001, with a core diameter of $1 \mathrm{~mm}$, a diameter with a jacket of $2.2 \mathrm{~mm}$, a reflective index of 1.49 , and a numerical aperture of 0.50. As shown in Equation (1), the displacement was $73 \mathrm{~mm}$ when the elbow was bent at $130^{\circ}$. Therefore, we set the sliding distance of the reflective surface to $73 \mathrm{~mm}$. The fiber-optic sensor was adjusted to the MEGA mode with a power of 41 . The target resolution of the elbow angle sensor was $4^{\circ}$. According to the 
experimental results presented in Section 2.2, $Q$ was 0.324 . Therefore, $K_{W_{-} \min }>$ $0.01 /(4 * Q)$ was 0.008 . Preliminary tests showed that the slope of the output curve of the sensor was larger than 0.008 when the optical fiber spacing was greater than $15 \mathrm{~mm}$. In our design, the distance between the two fibers was $15 \mathrm{~mm}$.

A similar test, as described in Section 4.1.1, was conducted to investigate the displacement sensing performance of the designed sensor. As shown in Figure 13 (d), the data obtained from multiple measurements indicated good repeatability. The MATLAB function fitting toolbox was used to perform Gaussian fitting of the average data, and the fitting curve in Figure 13 (e) was obtained $\left(R^{2}=1, R M E S=0.006997\right)$. Figure 13 (f) shows that the slope was between -0.0103 and -0.09238 when the displacement was in the range of $15.37-88 \mathrm{~mm}$. [figure 13 near here]

\subsection{Sensor calibration}

In this section, the process of sensor calibration for monitoring the wrist joint angle and the elbow joint angle is described. During the calibration process, the relationship between the joint angle and the sensor output voltage was acquired.

\subsubsection{Sensor calibration for monitoring the wrist joint motion}

Figure 14 (a) shows a user wearing the sensor. The sensor was attached to the subject's forearm using Velcro and fabric. One end of the non-retractable cable was attached to the sensor's sliding part, and the other end is attached to the subject's skin using the cable fixture fabricated by a 3D printer. The cable was restricted to move along the skin of the wrist using suture knots on the elastic fabric.

Figure 14 (b) shows the calibration experimental set-up. A digital protractor, (5414-200 Sanhe Measuring Instrument Co. LTD Zhejiang Province, China) was employed here for benchmarking. The digital protractor has a measurement range of 0-360 degrees with an accuracy of 
\pm 0.1 degrees. In the experiment, the designed sensor was attached to the user's forearm first.

Then one leg of the digital protractor was fixed to the user's hand and the other leg of the digital protractor was fixed to the user's forearm with tapes. The rotation center of the protractor was adjusted to align with the rotation center of the wrist. During the test, the subjects were asked to bend their wrists towards the palm from $0^{\circ}$ to $60^{\circ}$ and move back to $0^{\circ}$, with an interval of $3^{\circ}$ with the help of the protractor. The output voltage of the sensor was recorded at each angle interval. A digital protractor was used to measure the joint angle. The test was repeated 3 times. The angle of wrist flexion and the output voltage were compared in figure 14(c), and the relationships can be described as

$$
\begin{cases}V_{w f}=-0.02962 x_{w}+4.83 & (R-\text { square: } 0.9802) \\ V_{w r}=-0.0296 x_{w}+4.82 & (R-\text { square: } 0.9878)\end{cases}
$$

where $x_{w}$ is the wrist joint angle. $V_{w f}$ is the output voltage during the process of palm flexion (wrist moves from the position represented by the dashed line to the position represented by the solid line in figure1(b)), and $V_{w r}$ is the output voltage during the returning motion (wrist moves from the position represented by the solid line to the position represented by the dashed line in figure1(b)). The results showed that the maximum measurable palm flexion of the wrist joint was $60^{\circ}$. The sensitivity was $0.0296 \mathrm{~V} /{ }^{\circ}$. The resolution of the sensor was $0.338^{\circ}$, satisfying the target of $1^{\circ}$. The repeatability error was $8.23 \%$ when moving forward and $8.05 \%$ when moving backward. The linearity error was $3.73 \%$. [figure 14 near here]

\subsubsection{Sensor calibration for monitoring the elbow joint motion}

A similar experimental set-up was used in the sensor calibration for monitoring the elbow joint motion. In the experiment, the designed sensor was attached to the user's arm first. Then one leg of the digital protractor was fixed to the user's forearm and the other leg of the digital protractor was fixed to the user's upper arm with tapes. The rotation center of the protractor was adjusted to align with the rotation center of the 
elbow. Figure 15 (a) shows the calibration experimental set-up. During the test, the subjects were asked to bend their elbow from $0^{\circ}$ to $120^{\circ}$ and move back to $0^{\circ}$ with an interval of $3^{\circ}$ with the help of the protractor. The output voltage of the sensor was recorded at each angle interval. A digital protractor was used to measure the joint angle. The test was repeated 3 times. The experimental results are shown in Figure 15 (b). The relationships between the inputs and outputs can be described as

$$
\begin{cases}V_{e f}=2.197 * \exp \left(-0.03665 * x_{e}\right)+2.498 & (R-\text { square: } 0.9762) \\ V_{e r}=3.046 * \exp \left(-0.02215 * x_{e}\right)+2.211 & (R-\text { square: } 0.9699)\end{cases}
$$

where $x_{e}$ is the elbow angle. $V_{e f}$ is the output voltage during the elbow flexion motion (forearm moves from the position represented by the dashed line to the position represented by the solid line in figure1(a)), and $V_{e r}$ is the output voltage during the returning motion (forearm moves from the position represented by the solid line to the position represented by the dashed line in figure1(a)). The results show that the sensitivity while moving forward was $0.0028 \mathrm{~V} /{ }^{\circ}$, the sensitivity while moving backward was $0.0054 \mathrm{~V} /{ }^{\circ}$, and the resolution of the sensor was $3.6^{\circ}$ satisfying the target of $4^{\circ}$. The repeatability error was $11.08 \%$ when moving forward and $21.54 \%$ when moving backward. The hysteresis was $28.56 \%$. [figure 15 near here]

\subsubsection{Discussion}

In the calibration experiments, the elbow motion monitoring sensor had larger measurement errors than the wrist motion monitoring sensor. The measurement errors might have been caused by the following factors.

(1) Since we used elastic fabric, the elbow sensor was not anchored to the arm of the user firmly at all times, which had a significant influence on the hysteresis. The use of an active anchor structure [36] may provide more rigidity. 
(2) The rotation center of the protractor did not coincide with the rotation center of the elbow joint. In addition, the protractor was in direct contact with the joint, which might have resulted in frictional forces in different directions of the skin surface during the forward and reverse movements.

(3) The sliding part was in a clearance fit with the tube, and the position of the reflective surface was affected by friction during the movement.

Human wrist motions include palm flexion, dorsiflexion, radial deviation, and ulnar deviation. In this study, only the palm flexion of the wrist was considered. Since the range of palm flexion is larger than that of dorsiflexion, the sensor can also be attached to the other side of the forearm to monitor dorsiflexion. In the future, we will focus on developing a sensor with the ability to measure wrist dorsiflexion, radial deviation, and ulnar deviation.

In this study, a digital protractor was used for benchmarking of the proposed sensor. However, in the calibration process, even with the help of the digital protractor, it was difficult for the user to move to the required positions accurately, which might have affected the accuracy of the calibration. In the future, a VICON vision measurement system, which is more accurate and is able to be used in continues measurements, will be utilized as a standard sensor to measure the joint angle for benchmarking the proposed sensor.

\section{Conclusion}

In this paper, a wearable intensity-modulated optical fiber sensor for monitoring elbow and wrist joint movement was presented. A mathematical model of the sensing principle was established, and the potential influencing factors of the sensor were investigated. Two prototypes for elbow and wrist joint angle monitoring were fabricated and validated. The experimental results showed that for wrist motion monitoring, the 
maximum measurable palm flexion, sensitivity, resolution, and the linearity error were $60^{\circ}, 0.0296 \mathrm{~V} /{ }^{\circ}, 0.338^{\circ}$, and $3.73 \%$, respectively. For elbow motion monitoring, the maximum measurable flexion, the sensitivity during forward movement, the sensitivity during backward movement, the resolution, and the hysteresis error were $120^{\circ}, 0.0028$ $\mathrm{V} /{ }^{\circ}, 0.0054 \mathrm{~V} /{ }^{\circ}, 3.6^{\circ}$, and $28.56 \%$, respectively. The proposed sensor is not affected by electromagnetic interference and is lightweight. It has the potential to be used for rehabilitation purposes and the monitoring of human joint angles while performing reaching tasks, which are the most common upper-limb human gestures. In the future, the proposed sensor will be improved by adding an anchor structure for better fixation to the arm, adding sensing ability for wrist dorsiflexion, radial deviation, and ulnar deviation.

\section{Acknowledgment}

We thank the participants of the experiments.

\section{Funding details}

This work was partially funded by the National Natural Science Foundation of China under Grant [51975451]; RSE - NSFC Joint Project under Grant [51911530243]; the China Postdoctoral Science Foundation under Grant [2019M653586]; the Natural Science Foundation of Shaanxi Province of China under Grant [2019JQ-332]; the State Key Laboratory of Robotics under Grant [2019-O08], and the Fundamental Research Funds for the Central Universities under Grant [xzy012019012].

\section{Reference}

[1] C. Echalier et al., "Variability of shoulder girdle proprioception in 44 healthy volunteers," Orthopaedics \& Traumatology: Surgery \& Research, pp. 1-5, 2019. 
[2] Polygerinos P, Lyne S, Wang Z, Nicolini LF, Mosadegh B, Whitesides GM, and Walsh CJ, “Towards a Soft Pneumatic Glove for Hand Rehabilitation," in Proceedings on IEEE/RSJ International Conference on Intelligent Robots and Systems, 2013, pp.1512-1517.

[3] P. Polygerinos, Z. Wang, K. Galloway, et al., "Soft robotic glove for combined assistance and at-home rehabilitation," Robotics \& Autonomous Systems, pp. 135-143, 2015.

[4] Yap HK, Goh JCH, and Yeow RCH, "Design and Characterization of Soft Actuator for Hand Rehabilitation Application," in 6th European Conference of the International Federation for Medical and Biological Engineering, 2015, pp.367-370.

[5] KC. Galloway, P. Polygerinos, CJ. Walsh, and RJ. Wood, "Mechanically programmable bend radius for fiber-reinforced soft actuators," in IEEE 16th International Conference on Advanced Robotics [ICAR], 2013, pp.1-6.

[6] D. Latz et al, "Doctor, when can I drive? — Range of motion of the knee while driving a car," The Knee, pp. 33-39, 2018.

[7] D. Latz et al, "Influence of Wrist Position on the Metacarpophalangeal Joint Motion of the Index Through Small Finger," Surgery Article., vol. 14, no.2, pp.259-263, 2017.

[8] Mahvash, Mohsen, and P. E. Dupont, "Stiffness Control of Surgical Continuum Manipulators." IEEE Transactions on Robotics 27.2[2011]:334-345.

[9] Melzer, Michael, et al. "Wearable Magnetic Field Sensors for Flexible Electronics [pages 1274-1280]." Advanced Materials 27.7[2015]:1274-1280.

[10] R. Baker, “Gait analysis methods in rehabilitation,” J. Neuroeng. Rehabil., 2006.

[11] C. Hu, Y. Ge, and Y. Chen, "Measurement System for human joint range of motion," Chinese Journal of Medical Physics, vol. 33, no.1, pp. 34-38, 2016.

[12] W. Wang, Y. Guo, H. Gao, H. Li, T. Zheng, and L. Lin, "Measuring joint range of motion and evaluating measured results based on depth images," Chinese J. Med. Phys., no. 3, pp. 262-269, 2016.

[13] M. Amjadi, K. Kyung, I. Park, and M. Sitti, "Stretchable, Skin-Mountable, and Wearable Strain Sensors and Their Potential Applications: A Review," Advanced Functional Materials, vol. 26, no.11, pp. 1679-1698, 2016.

[14] A. Nag, S. C. Mukhopadhyay, and J. Kosel, “Wearable Flexible Sensors: A Review,” IEEE Sensors Journal, vol. 17, no.13, pp. 3949-3960, 2017.

[15] T. Yamada, et al., “A stretchable carbon nanotube strain sensor for human-motion detection,” NATURE NANOTECHNOLOGY, 2011, pp. 296-301.

[16] Y. Li, et al., "Highly Flexible Strain Sensor from Tissue Paper for Wearable Electronics," ACS Sustainable Chemistry \& Engineering, pp.4288-4295, 2016.

[17] J. Jeon, and T. Ha, "Waterproof Electronic-Bandage with Tunable Sensitivity for Wearable Strain Sensors," ACS Applied Materials \& Interfaces, pp. 2866-2871, 2016.

[18] P. T. Gibbs et al., "Wearable Conductive Fiber Sensors for Multi-Axis Human Joint Angle Measurements," J. Neuroeng. Rehabil., 2005.

[19] M. Tesconi et al., "Wearable sensorized system for analyzing the lower limb movement during rowing activity," IEEE International Symposium on Industrial Electronics, 2007.

[20] Yao, Shanshan, and Y. Zhu, "Wearable multifunctional sensors using printed stretchable conductors made of silver nanowires." Nanoscale, vol. 6, no. 4, pp. 2345-2352, 2014 
[21] J. Zubia and J. Arrue, "Plastic optical fibers: An introduction to their technological processes and applications," Opt. Fiber Technol., 2001.

[22] L. Bilro, N. Alberto, J. L. Pinto, and R. Nogueira, “Optical sensors based on plastic fibers,” Sensors [Switzerland], vol. 12, no. 9, pp. 12184-12207, 2012.

[23] M. A. Zawawi, S. O\&apos; Keffe, and E. Lewis, "Intensity-modulated fiber optic sensor for health monitoring applications: a comparative review," Sens. Rev., vol. 33, no. 1, pp. 57-67, 2013.

[24] L. Cherbi, M. Mehenni, and R. Aksas, "Conception and realization of an angular optical sensor," Microw. Opt. Technol. Lett., 2004

[25] A. S. Silva, A. Catarino, M. V. Correia, and O. Frazão, "Design and characterization of a wearable macrobending fiber optic sensor for human joint angle determination," Opt. Eng., vol. 52, no. 12, p. 126106, 2013.

[26] P. Puangmali, K. Althoefer, and L. D. Seneviratne, "Mathematical Modeling of Intensity-Modulated Bent-Tip Optical Fiber Displacement Sensors," IEEE Trans. Instrum. Meas., vol. 59, no. 2, pp. 283-291, Feb. 2010.

[27] J. B. Faria, “A theoretical analysis of the bifurcated fiber bundle displacement sensor," IEEE Trans. Instrum. Meas., vol. 47, no. 3, pp. 742-747, 1998.

[28] H. A. Rahman, N. M. Isa, and S. W. Harun, "Modeling the Concentric Fiber Bundle Displacement Sensor Using a QuasiGaussian Beam Approach,” IEEE Sensors Journal, vol. 15, no. 9, pp. 4777-4781, 2015.

[29] Yohan Noh, et al.,"Three-Axis Fiber-Optic Body Force Sensor for Flexible Manipulators." IEEE Sensors Journal vol. 16, no. 6, pp. 1641-1651, 2016

[30] Sargeant, Ramon, L. D. Seneviratne, and K. Althoefer. "A 2-Axis Optical Force-Torque Fingertip Sensor for Dexterous Grasping Using Linear Polarizers." IEEE Transactions on Instrumentation and Measurement, vol. 61. no. 12, pp. 3363-3377, 2012 .

[31] Xie, Hui , et al. "A Fiber-Optics-Based Body Contact Sensor for a Flexible Manipulator." IEEE Sensors Journal vol.15, no.6, pp. 3543-3550, 2015

[32] Liu, Hongbin , J. Back, and K. Althoefer . "Feasibility Study- Novel Optical Soft Tactile Array Sensing for Minimally Invasive Surgery." 2015 IEEE/RSJ International Conference on Intelligent Robots and Systems [IROS] IEEE, 2015.

[33] J. E. Nitschke, C. L. Nattrass, P. B. Disler, M. J. Chou, and K. T. Ooi, "Reliability of the American Medical Association guides' model for measuring spinal range of motion. Its implication for whole-person impairment rating.," Spine [Phila. Pa. 1976]., 1999.

[34] Z. Zhao, et al., "Modulation function of the reflective optical fiber sensor for specular and diffuse reflection," Optical Engineer, vol. 33, no. 9, pp. 2986-2991, 1994.

[35] H. Wang, "Effects of fiber geometry on the modulation function of a reflective intensity modulation sensor," Journal of Modern Optics, vol. 43, no. 11, pp. 2355-2366, 1996.

[36] H. Choi, et al., "Exo-Wrist: A Soft Tendon-Driven Wrist-Wearable Robot With Active Anchor for Dart-Throwing Motion in Hemiplegic Patients,” IEEE Robotics and Automation Letters, vol. 4, pp. 4499 - 4506, 2019. 


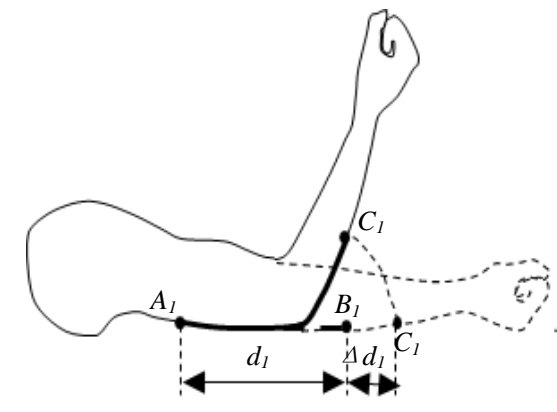

(a)

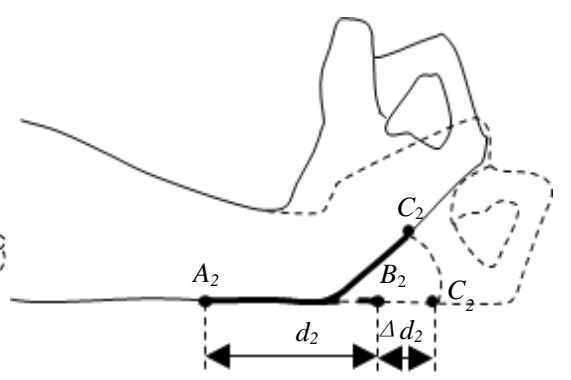

(b)

Figure 1. Joint angle sensing principle: (a) the elbow angle change can be measured by monitoring the displacement $\Delta d_{l}$, and (b) the wrist angle change can be measured by monitoring the displacement $\Delta d_{2}$.

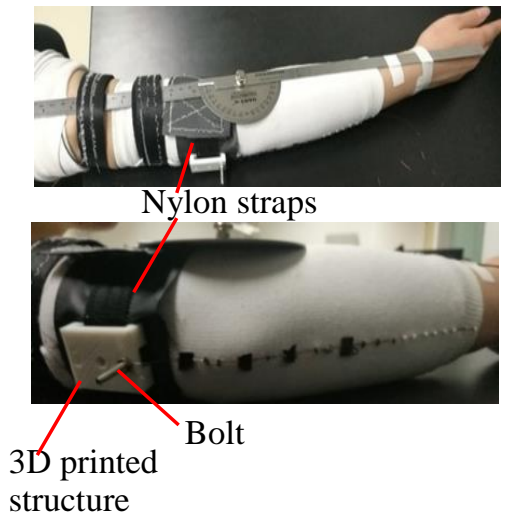

structure
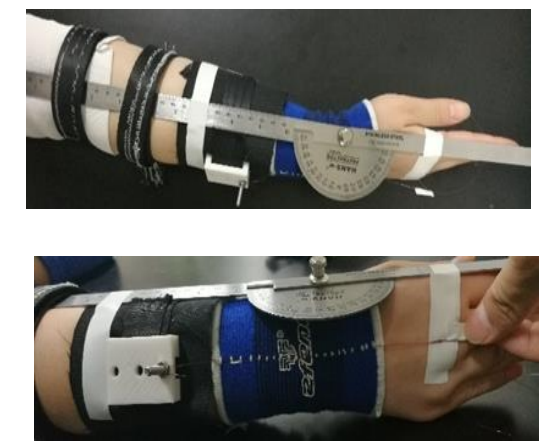

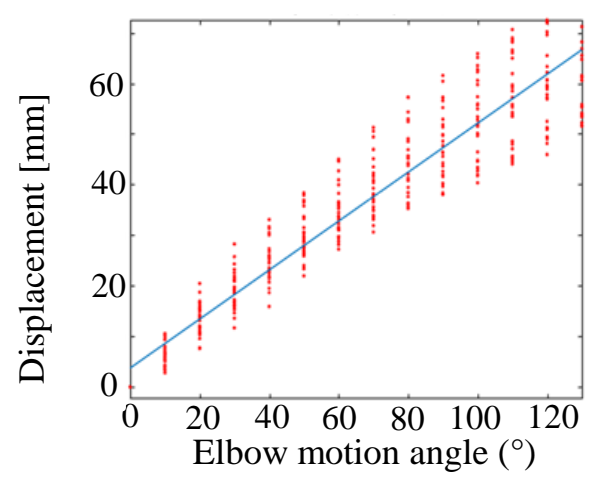

(a)

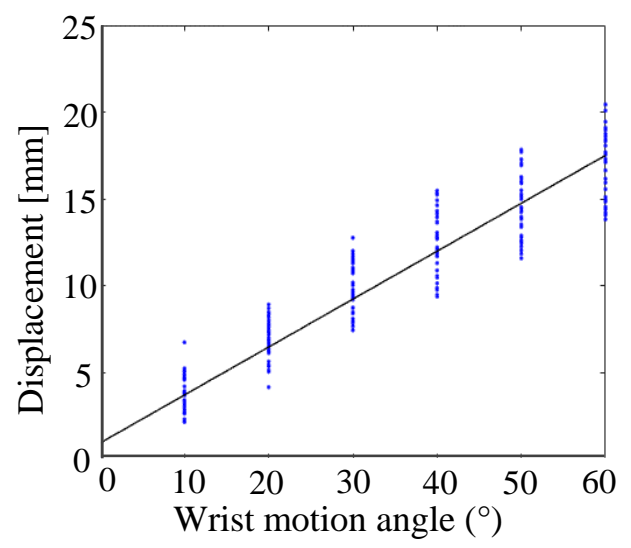

(b)

Figure 2. The relationship between (a) the elbow motion angle and the displacement and (b) the palm flexion angle and the displacement. The experimental setups are shown in the photos on the left, and the experimental results are shown in the graphs on the right. 


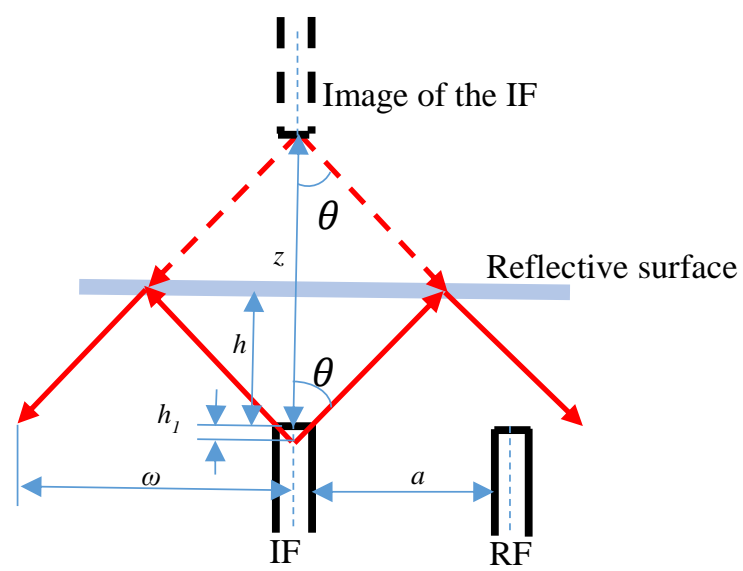

Figure 3. Principle of the reflective intensity-modulated fiber-optic sensor.

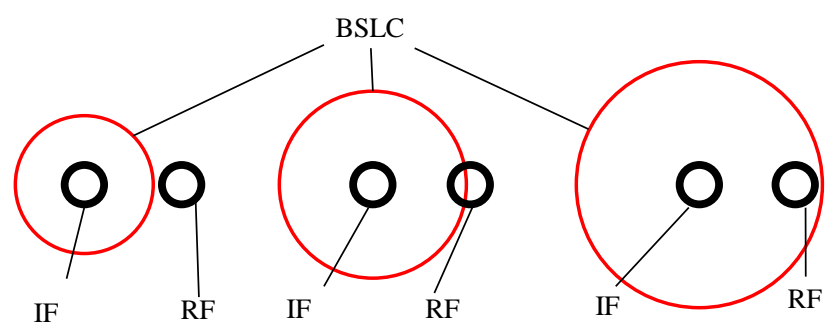

Figure 4 Diagram of the light intensity analysis of the RF for different positions of the reflective surface.

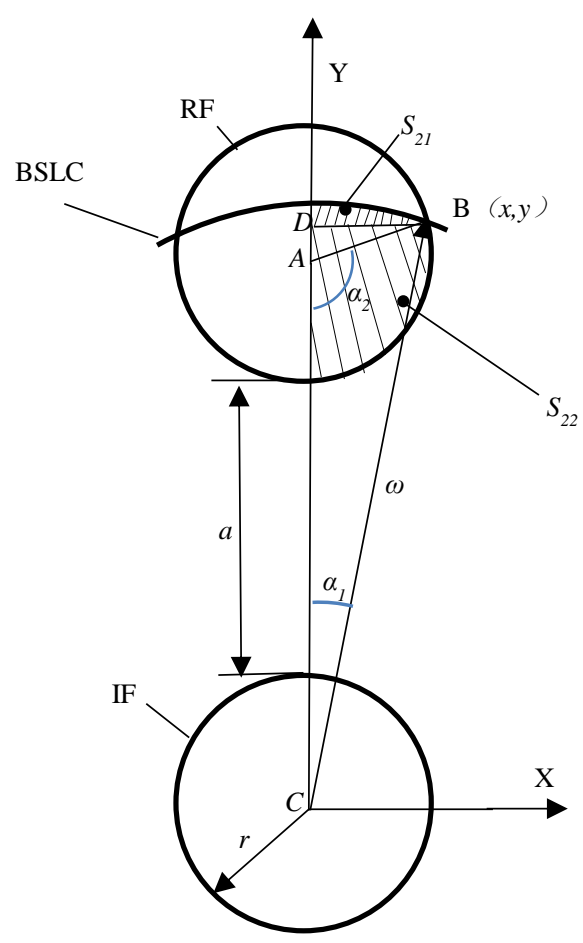

Figure 5 Schematic diagram of the calculation of the overlap area. 


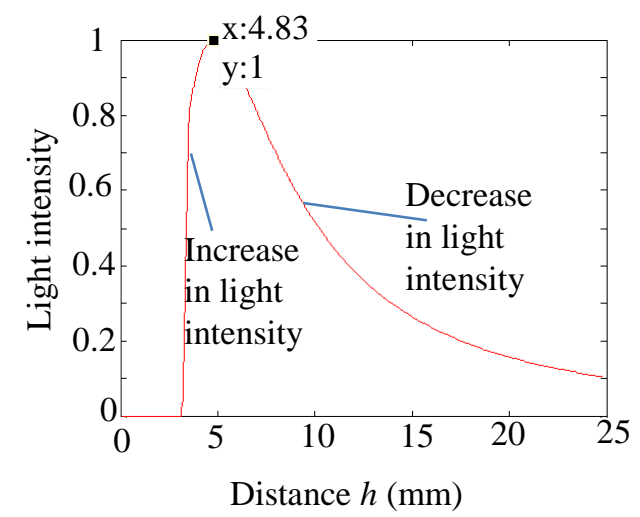

rigure o ine typıcal response or a renıctive intensıly-moaulated Iıber-optic sensor.

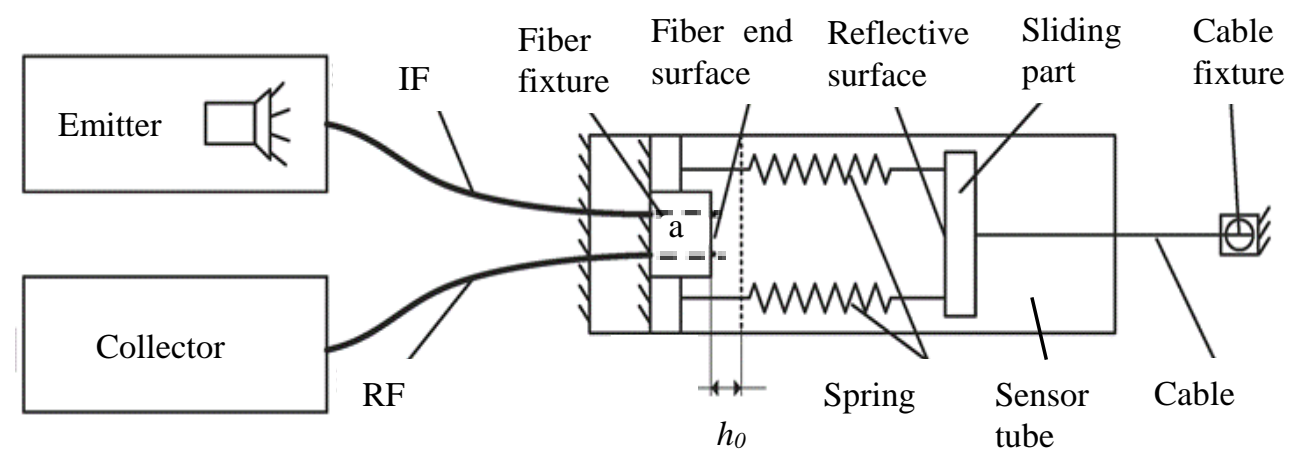

Figure 7 Diagram of the reflective intensity-modulated sensor. 

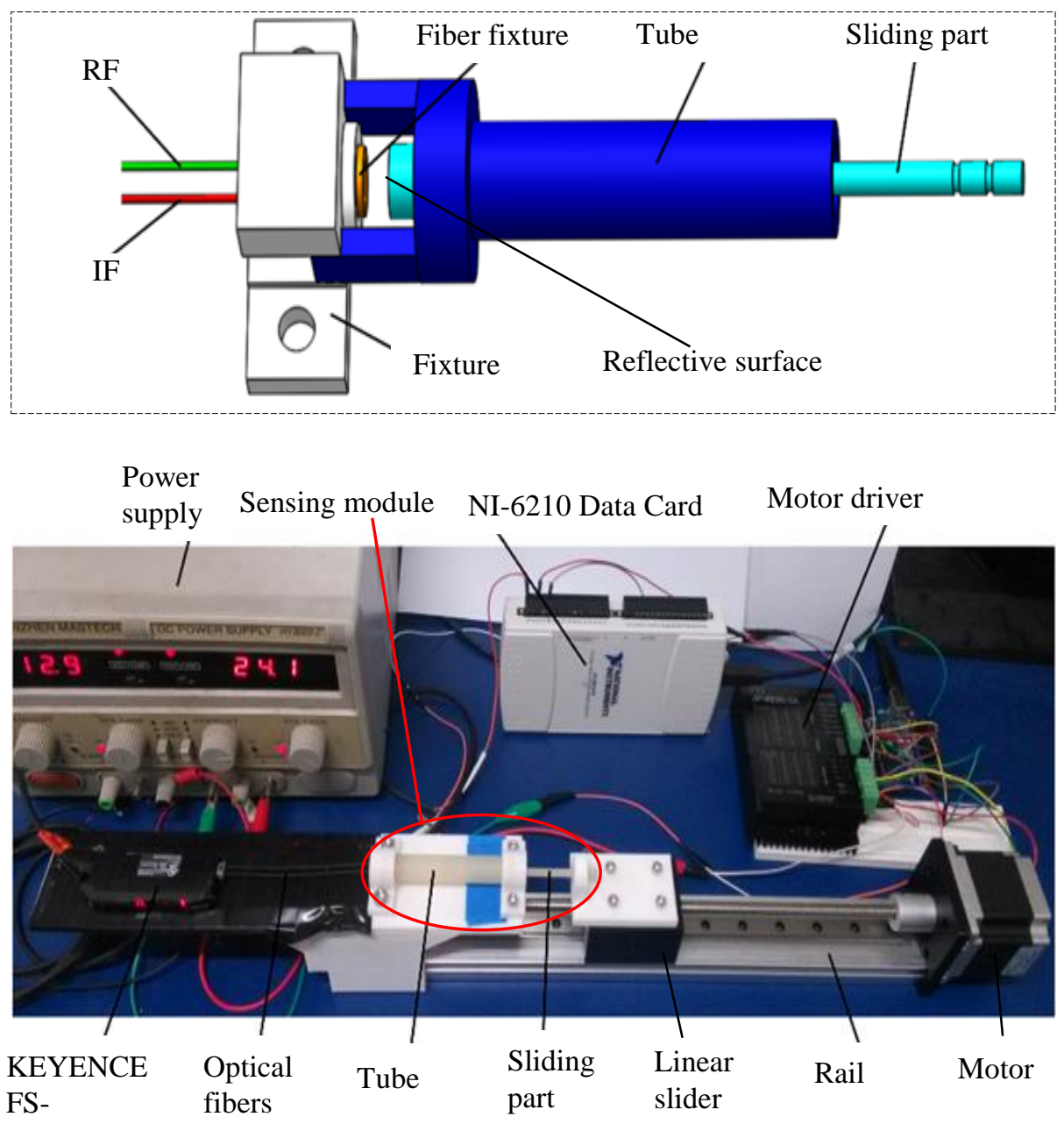

N11MN

Figure 8 Experimental setup for the displacement measurements.

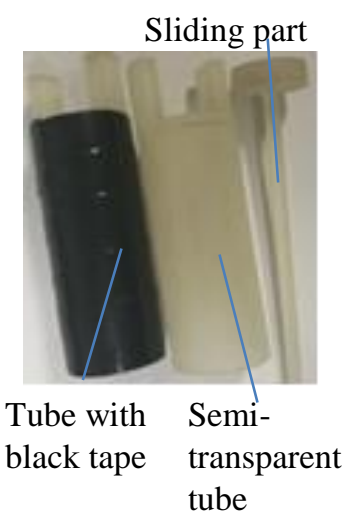

(a)

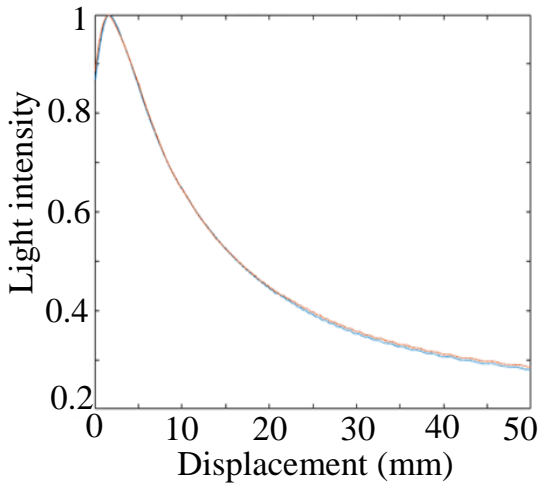

(b)

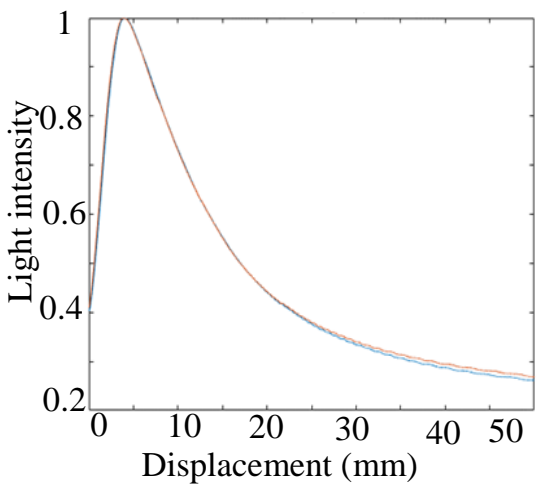

(c)

Figure 9 (a) The tube and sliding part used in the experiment to determine the influence of the tube's light transmittance on the light intensity; the relationship between the light 
intensity and displacement for a tube diameter of (b) $19.57 \mathrm{~mm}$ and (c) $15.74 \mathrm{~mm}$. The red line represents the results of the tube without black tape, and the blue line represents the results for the tube with the black tape.

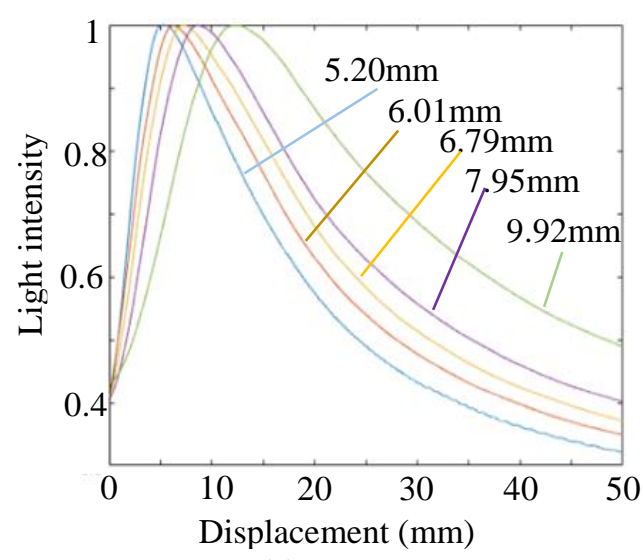

(a)

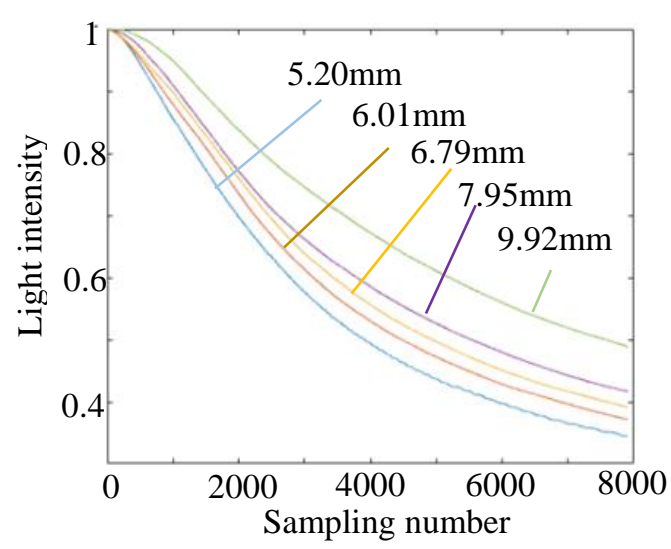

(b)

Figure 10 The effect of the distance between the IF and RF on the light intensity: (a) initial data for 5 sets of experiments, (b) details of the area of decrease of the curve.

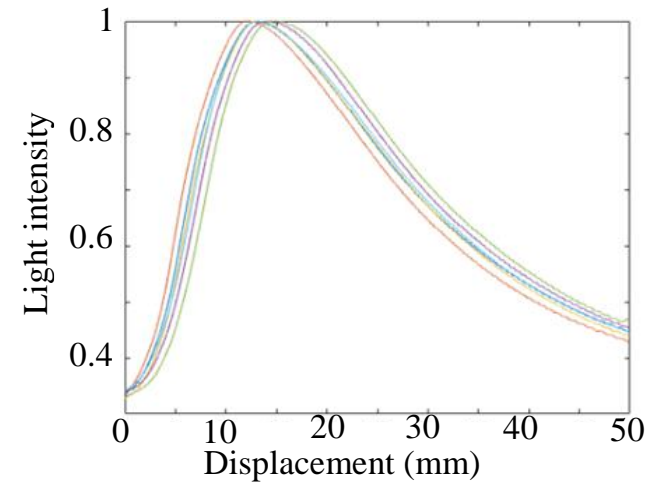

(a)

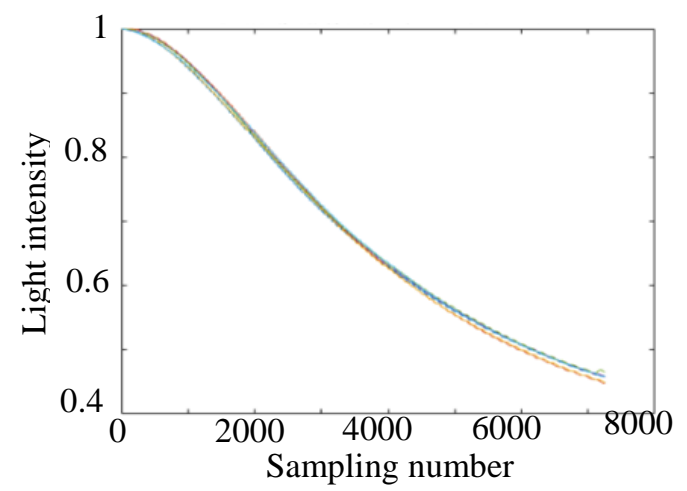

(b)

Figure 11 The effect of the tilt angle of the reflective surface on the light intensity: (a) initial data; (b) details of the area of decrease of the curve. 


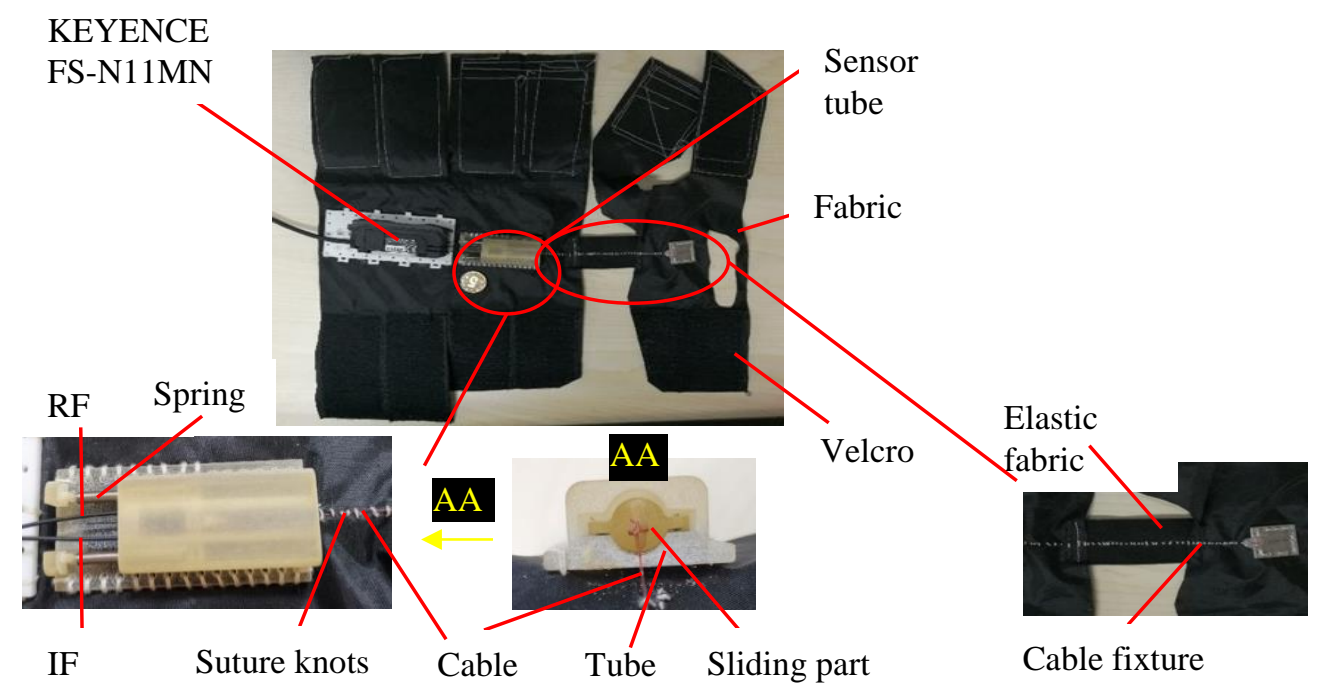

Figure 12 The prototype of the reflective intensity-modulated sensor for measuring the wrist motion angle.

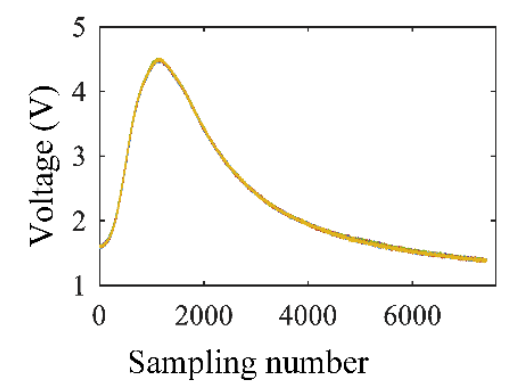

(a)

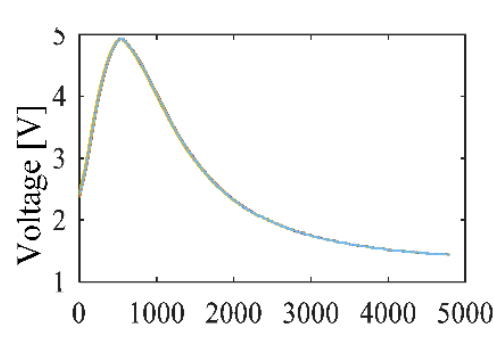

Sampling number

(d)

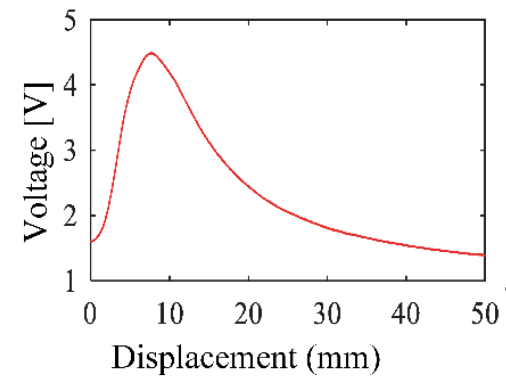

(b)

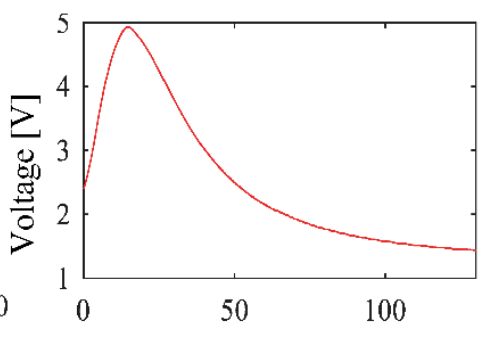

Displacement (mm)

(e)

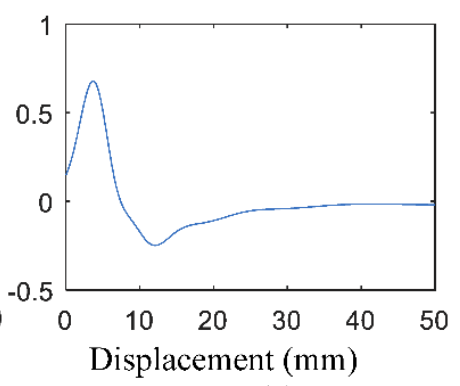

(c)

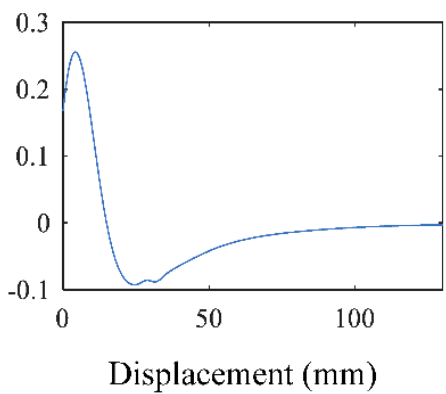

(f)

Figure 13 Displacement sensing performance of the sensor for monitoring wrist and elbow joint motion: initial data for the (a) wrist sensor and (d) elbow sensor, the fitted curve for the (b) wrist sensor and (e) elbow sensor, first derivative for the (c) wrist sensor and (f) elbow sensor. 


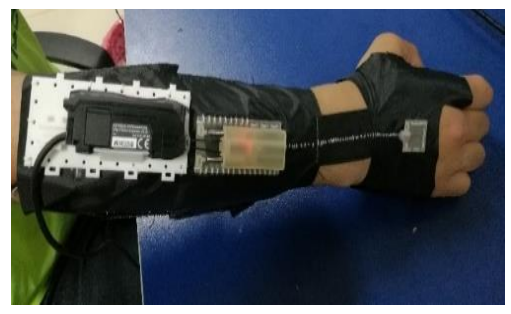

(a)

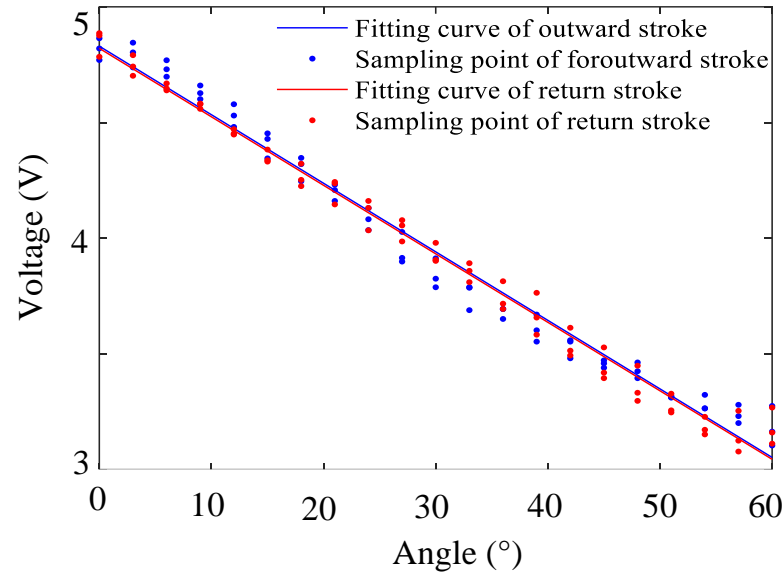

(c)

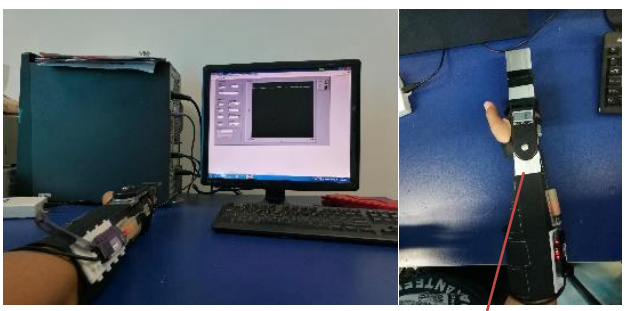

(b)

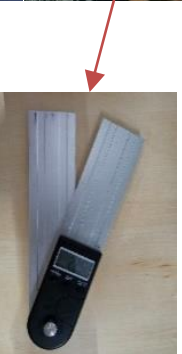

Digital protractor

Figure 14 (a) The sensor for monitoring the wrist joint motion worn by a user, (b) the calibration experimental set-up for the wrist sensor, and (c) the output voltage with increasing wrist palm flexion angle.

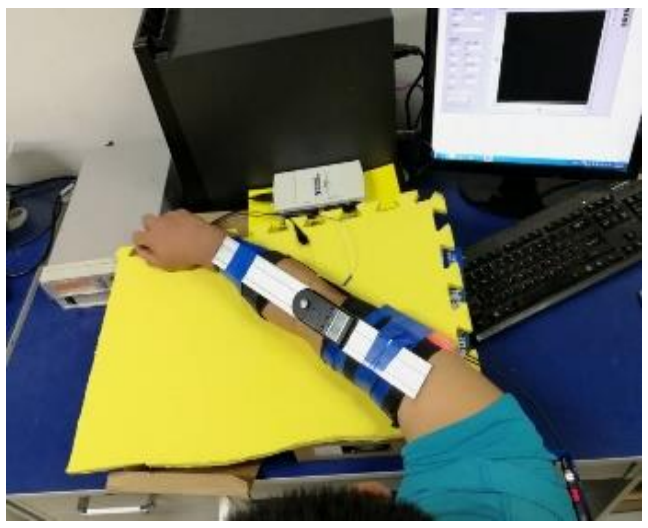

(a)

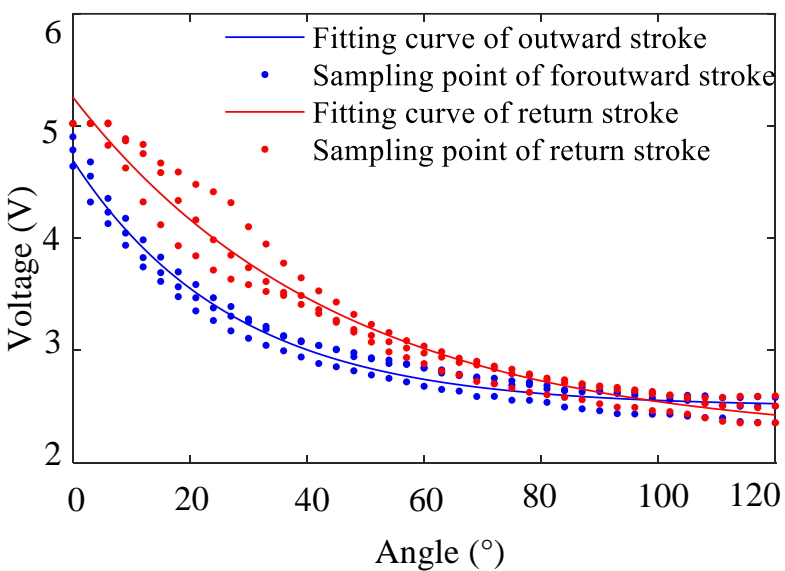

(b)

Figure 15 (a) Calibration experimental set-up for the elbow sensor and (b) the output voltage with increasing elbow flexion angle. 